Supporting Information

\title{
Semisynthetic and Biomolecular Hydrogen Evolution Catalysts
}

\author{
Banu Kandemir, Saikat Chakraborty, Yixing Guo, and Kara L. Bren*
}

Department of Chemistry, University of Rochester, Rochester NY 14627-0216

*e-mail: bren@chem.rochester.edu 

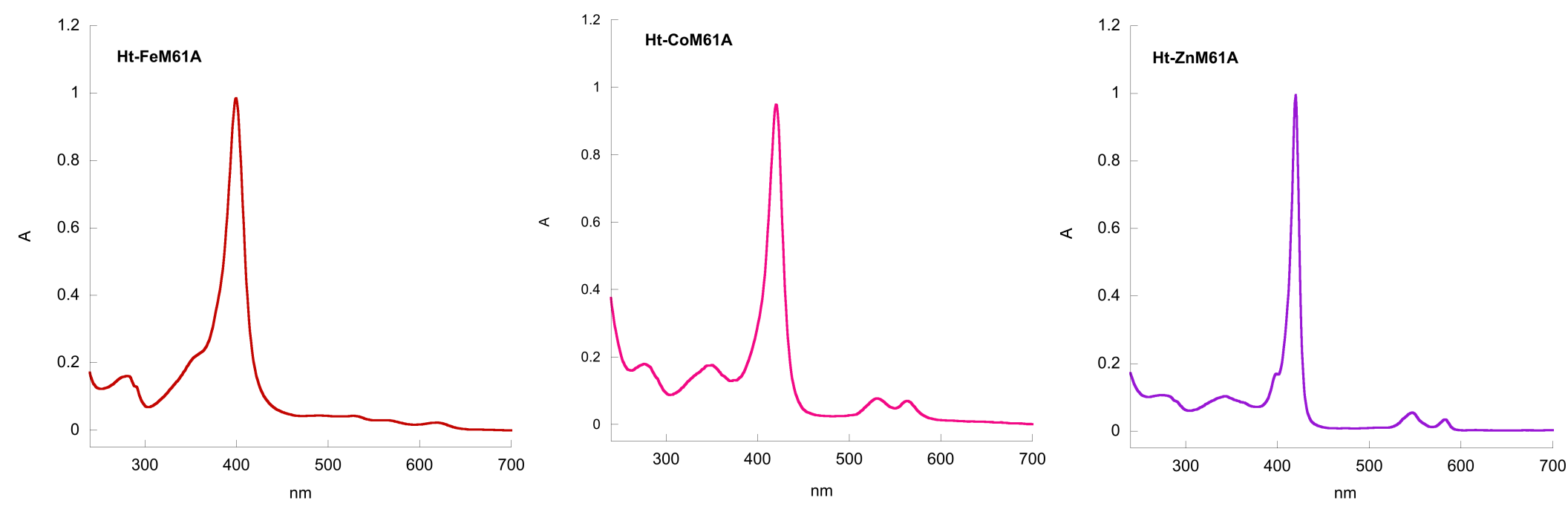

Figure S1. UV-vis absorption spectra of $H t-\mathrm{Fe}^{\mathrm{III}} \mathrm{M} 61 \mathrm{~A}$ (left), $H t-\mathrm{Co}{ }^{\mathrm{III}} \mathrm{M} 61 \mathrm{~A}$ (center), and $H t-\mathrm{Zn}{ }^{\mathrm{II}} \mathrm{M} 61 \mathrm{~A}$ (right). Samples were in 50 $\mathrm{mM} \mathrm{NaP}$ i $\mathrm{pH}$ 7.0. 


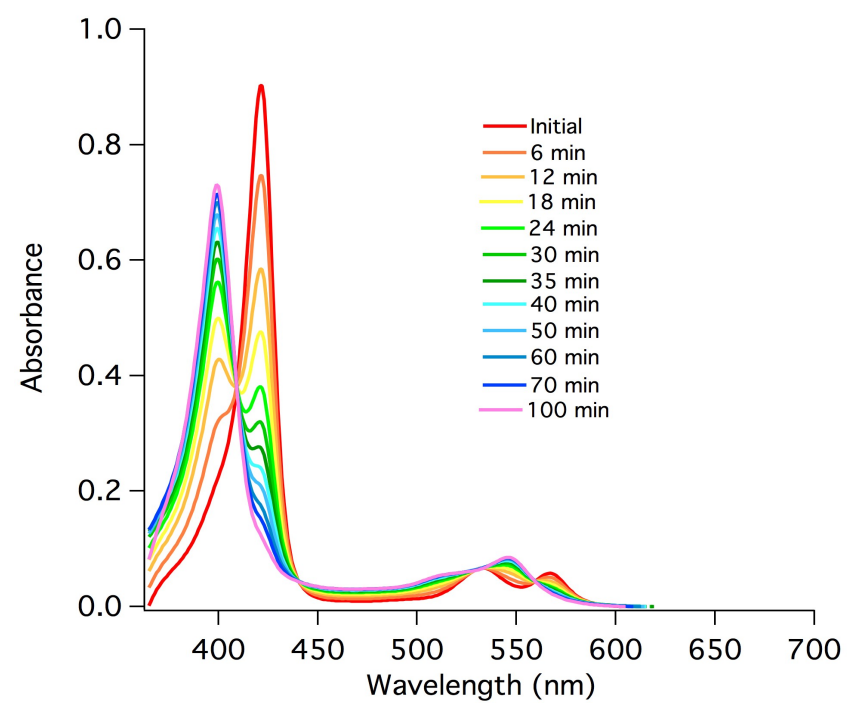

Figure S2. UV-Vis absorption spectra of $H t$-CoM61A in $50 \mathrm{mM} \mathrm{KP}$ i pH 7.0, in the presence of a 500 -fold excess of $\mathrm{Na}_{2} \mathrm{~S}_{2} \mathrm{O}_{4}$ as a function of time. The red "initial" spectrum is of the $\mathrm{Co}(\mathrm{III})$ protein, and the subsequent spectra demonstrate the slow reduction to the Co(II). The spectrum taken at 100 minutes is consistent with a Co(II) porphyrin.

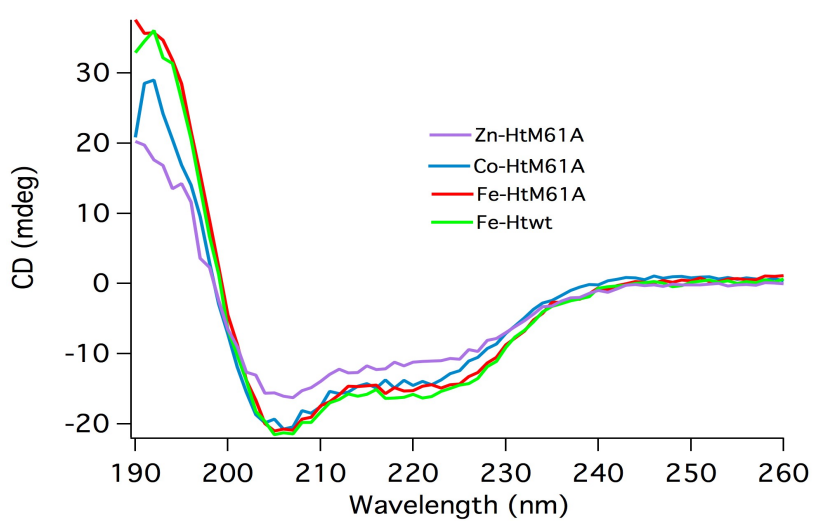

Figure S3. Circular dichroism spectra of $10 \mu \mathrm{M} H t$-CoM61A (blue), $H t$-ZnM61A (violet), $H t$-FeM61A (red) and $H t$-c-552 (green). Samples were in $50 \mathrm{mM} \mathrm{NaP}_{\mathrm{i}}, \mathrm{pH} 7.0$ 


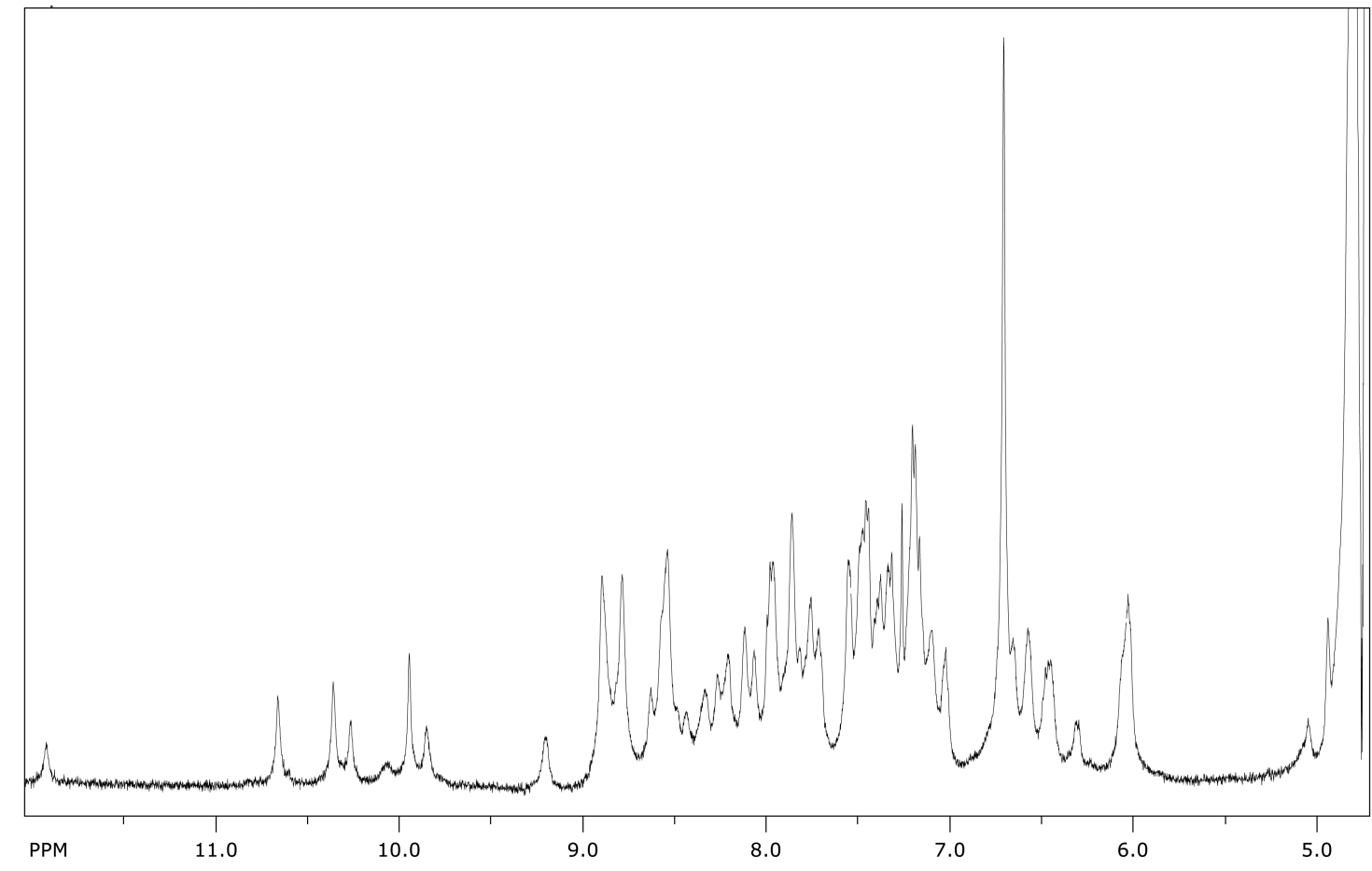

Figure S4. High-frequency region of $500-\mathrm{MHz}{ }^{1} \mathrm{H}$ NMR spectrum of $0.5 \mathrm{mM} \mathrm{Ht}$-CoM61A, $\mathrm{D}_{2} \mathrm{O}$, pH 5.0. The narrow line widths and peak dispersion indicate that the protein is folded with a well defined tertiary structure. 


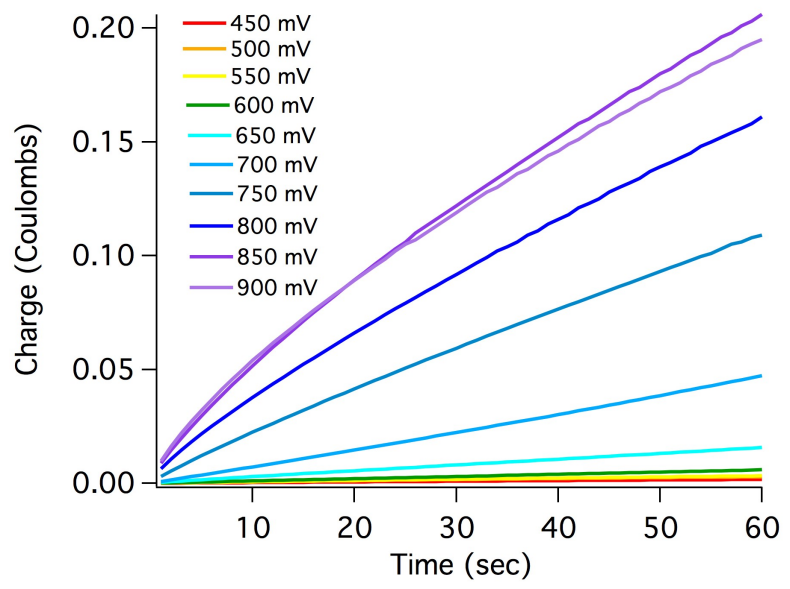

Figure S5. Ladder plot showing the effect of applied overpotential on the charge passed in 1-minute CPE experiments on $1.0 \mu \mathrm{M} \mathrm{Ht}$ CoM61A in $2 \mathrm{M} \mathrm{KP}_{\mathrm{i}}, \mathrm{pH} 7.0$. Overpotential was calculated using a potential for proton reduction of $-649 \mathrm{mV} \mathrm{vs} . \mathrm{Ag} / \mathrm{AgCl}(1 \mathrm{M} \mathrm{KCl})$ at $\mathrm{pH}$ 7.0.

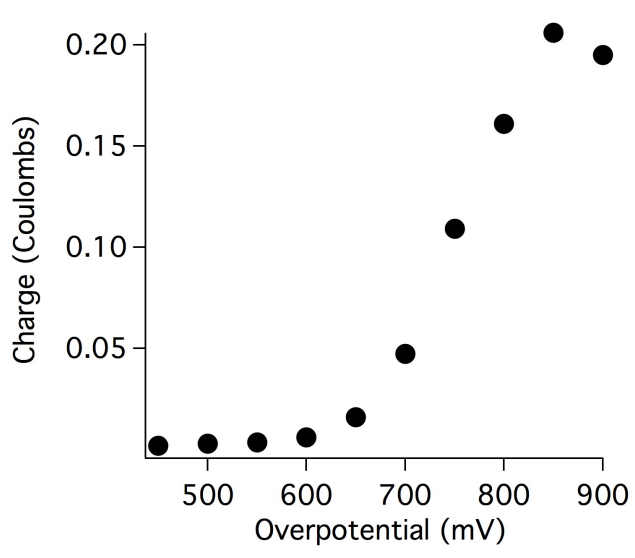

Figure S6. Plot of charge passed in 1-minute $\mathrm{CPE}$ of $H t$-CoM61A in $2 \mathrm{M} \mathrm{KP}$, pH 7.0 as a function of the applied overpotential (Figure S5 data). 


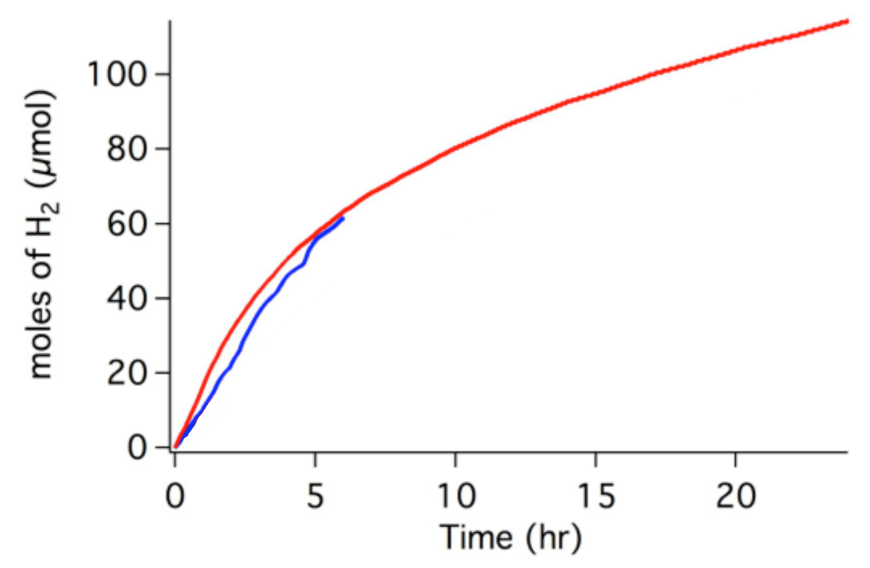

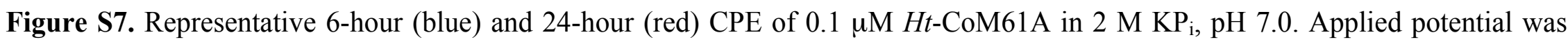
$-1.45 \mathrm{~V}$ vs $\mathrm{Ag} / \mathrm{AgCl}(1 \mathrm{M} \mathrm{KCl})$.

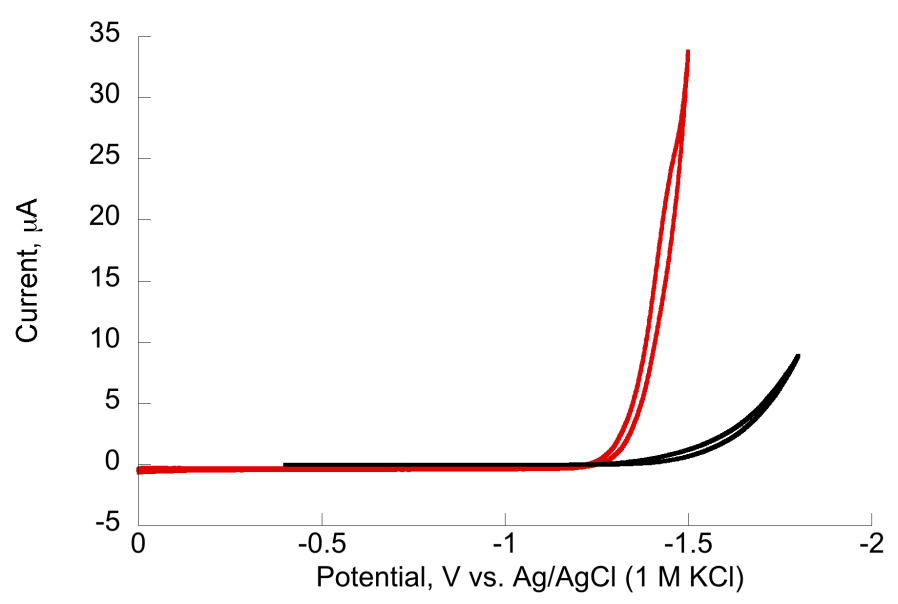

Figure S8. Cyclic voltammogram (100 mV/s) of $1.0 \mu \mathrm{M} \mathrm{Ht}$-CoM61A in $2 \mathrm{M} \mathrm{KP}$, pH 7.0 (red), and of buffer background (black). Voltage range of scan was limited to show onset potential. 


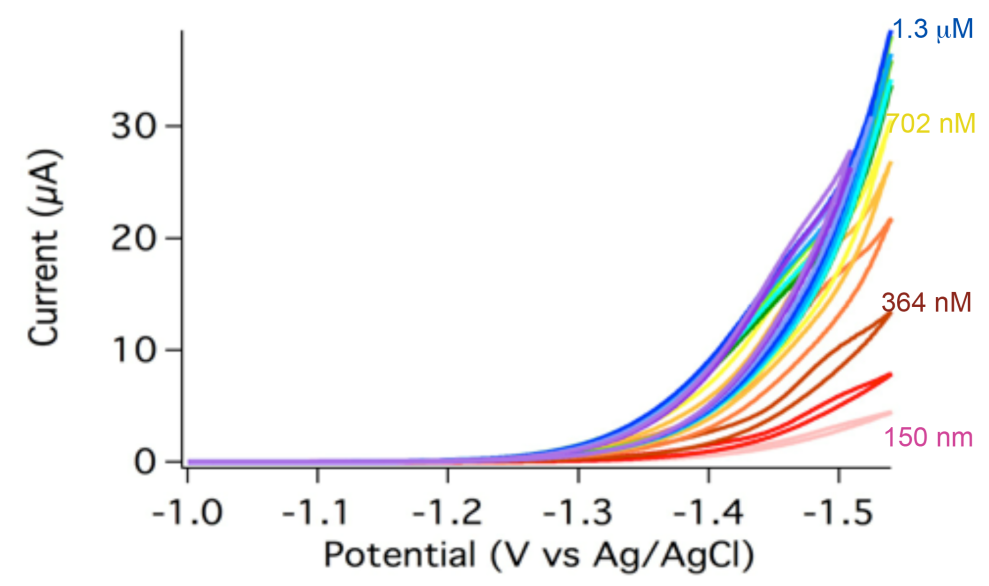

Figure S9. Cyclic voltammograms $(100 \mathrm{mV} / \mathrm{s})$ of varied concentrations (100 nM (pink) - $1.6 \mu \mathrm{M}$ (purple)) of $H t$-CoM61 A in $2 \mathrm{M}$ $\mathrm{KP}_{\mathrm{i}}, \mathrm{pH}$ 7.0. Selected scans are labeled, and peak current is plotted for all data in Figure S10.

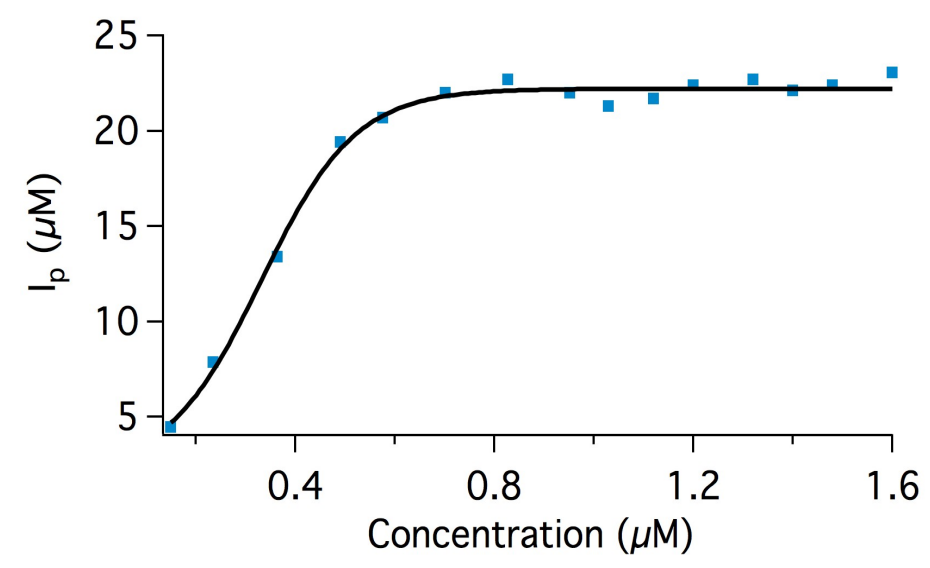

Figure S10. Plot of the peak current $\left(\mathrm{I}_{\mathrm{P}}\right)$ vs. $H t$-CoM61A concentration for the data in Figure S9. 


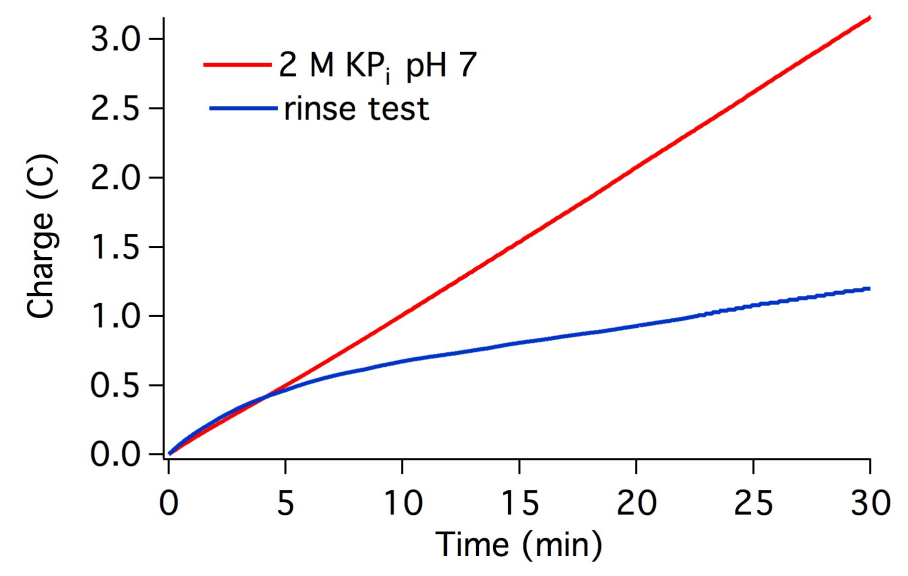

Figure S11. CPE $(-1.45 \mathrm{~V}$ vs. $\mathrm{Ag} / \mathrm{AgCl}(1 \mathrm{M} \mathrm{KCl}))$ of $1.0 \mu \mathrm{M} \mathrm{Ht}$-CoM61 A in $2 \mathrm{M} \mathrm{KP}_{\mathrm{i}}, \mathrm{pH} 7.0$ (red), and of $2 \mathrm{M} \mathrm{KP}$, $\mathrm{pH} 7.0$ after rinsing the electrode (blue).

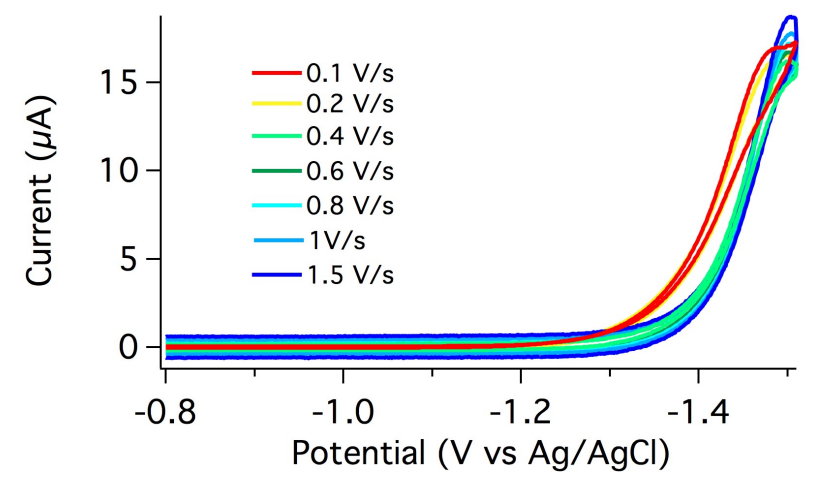

Figure S12. Cyclic voltammograms of $1.0 \mu \mathrm{MHt}$-CoM61A in $2 \mathrm{M} \mathrm{KP}_{\mathrm{i}}, \mathrm{pH} 7$ as a function of scan rate. 


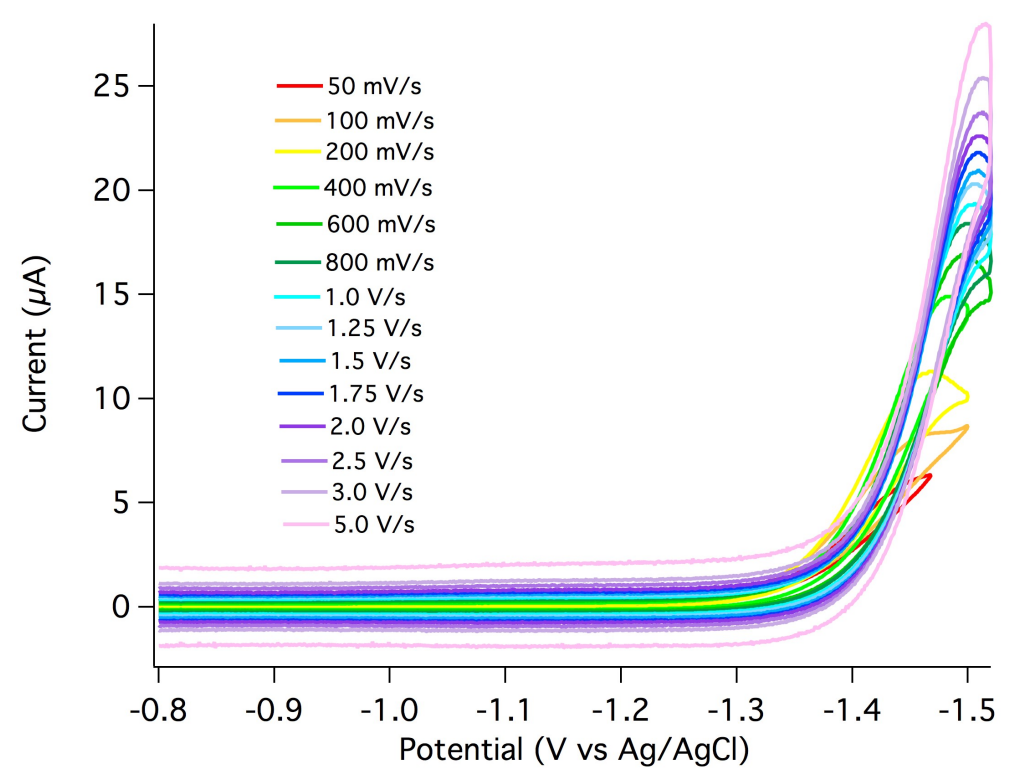

Figure S13. Cyclic voltammograms of $1.0 \mu \mathrm{M} \mathrm{Ht}$-CoM61A in $10 \mathrm{mM} \mathrm{KP}, 1 \mathrm{M} \mathrm{KNO}_{3} \mathrm{pH} 7$ as a function of scan rate.

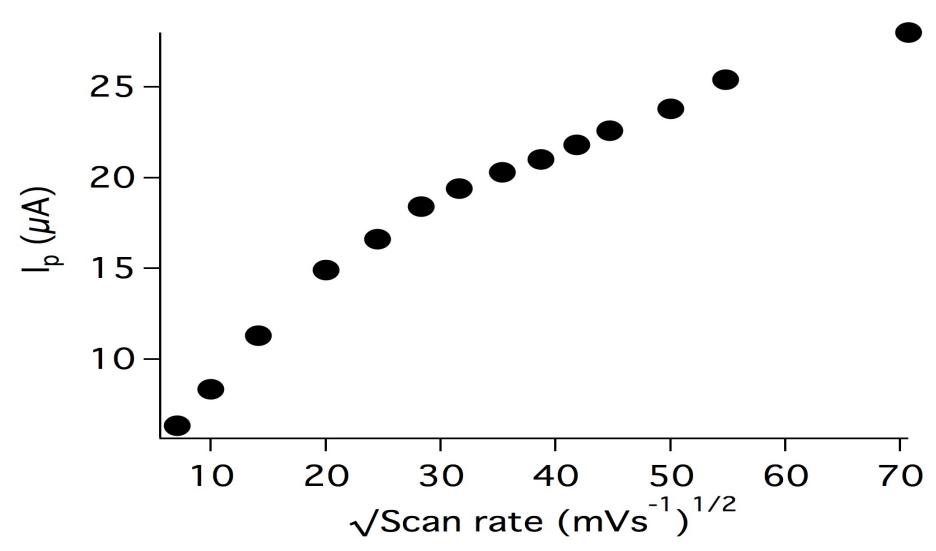

Figure S14. $I_{p}$ vs square root of scan rate for data in Figure S13. 
Table S1. Properties of Catalysts and Assemblies for Photocatalytic Production of $\mathrm{H}_{2}$ from Water

\begin{tabular}{|c|c|c|c|c|c|c|c|c|}
\hline Catalyst & Photosensitizer & $\begin{array}{l}\text { Catalyst- } \\
\text { photosensitizer } \\
\text { interaction }\end{array}$ & $\begin{array}{l}\text { Electron } \\
\text { donor }\end{array}$ & $\begin{array}{l}\text { Solution } \\
\text { conditions } \\
(\mathrm{pH})^{a}\end{array}$ & $\begin{array}{l}\text { TON (mol } \\
\mathrm{H}_{2} / \mathrm{mol}^{b} \\
\text { catalyst) }^{b}\end{array}$ & $\begin{array}{l}\text { Longevity } \\
\text { (hours) }^{c}\end{array}$ & Comments & Ref. \\
\hline cobaloxime & PSI & intramolecular & ascorbate & MES (6.3) & 5200 & 1.5 & $\begin{array}{l}\text { TON with respect to } \\
\text { PSI }\end{array}$ & 1 \\
\hline $\mathrm{Ni}\left(\mathrm{P}_{2} \mathrm{~N}_{2}\right)_{2}$ & PSI & intramolecular & ascorbate & MES (6.3) & 1870 & 3 & $\begin{array}{l}\text { TON with respect to } \\
\text { PSI }\end{array}$ & 2 \\
\hline Ni diphosphine & PSI & diffusion & ascorbate & MES (6.3) & 2825 & 4 & $\begin{array}{l}\text { TON with respect to } \\
\text { PSI }\end{array}$ & 2 \\
\hline cobaloxime & $\begin{array}{l}{\left[\mathrm{Ru}(\mathrm{bpy})_{3}\right]^{2+}} \\
\text { derivative }\end{array}$ & $\begin{array}{l}\text { Ru covalent binding } \\
\text { to C18; cobaloxime } \\
\text { nonspecifically } \\
\text { bound }\end{array}$ & ascorbate & MES (6.3) & $210 \pm 60$ & 6 & $\begin{array}{l}\text { TON with respect to } \\
{\left[\mathrm{Ru}(\mathrm{bpy})_{3}\right]^{2+}}\end{array}$ & 3 \\
\hline $\begin{array}{l}\text { myoglobin- } \\
\text { cobaloxime }\end{array}$ & $\begin{array}{l}\text { diamino- } \\
\text { fluorescein }\end{array}$ & diffusion & ascorbate & Tris-HCl (7) & 8.3 & -- & $\begin{array}{l}\text { TON with respect to } \\
\text { introduced Co center }\end{array}$ & 4 \\
\hline$[\mathrm{FeFe}]^{d}$ & {$\left[\mathrm{Ru}(\mathrm{bpy})_{3}\right]^{2+}$} & diffusion & ascorbate & citrate (4.5) & 84 & 2.3 & $\begin{array}{l}\text { Fe coordination to a } \\
\text { dithiol amino acid }\end{array}$ & 5 \\
\hline [FeFe] & {$[\mathrm{Ru}(\mathrm{bpy})(\mathrm{tpy})]^{2+}$} & intramolecular & ascorbate & $\begin{array}{l}\text { Tris- } \mathrm{HCl} \\
(8.5)\end{array}$ & 9 & 2 & $\begin{array}{l}\text { Diiron cluster } \\
\text { attached to Cys of } \\
\mathrm{CXXCH}\end{array}$ & 6 \\
\hline [FeFe] & {$\left[\mathrm{Ru}(\mathrm{bpy})_{3}\right]^{2+}$} & diffusion & ascorbate & $\begin{array}{l}\text { Tris- } \mathrm{HCl} \\
(4.7)\end{array}$ & 80 & 2 & $\begin{array}{l}\text { Diiron cluster } \\
\text { attached to Cys of } \\
\mathrm{CXXCH}\end{array}$ & 7 \\
\hline [FeFe]-nitrobindin & {$\left[\mathrm{Ru}(\mathrm{bpy})_{3}\right]^{2+}$} & diffusion & ascorbate & Tris-HCl (4) & 130 & 6 & $\begin{array}{l}\text { Diiron cluster } \\
\text { covalently attached } \\
\text { to nitrobindin }\end{array}$ & 8 \\
\hline $\begin{array}{l}\text { D. desulfuricans } \\
\text { Ni-rubredoxin }\end{array}$ & -- & -- & $\begin{array}{l}\text { methyl } \\
\text { viologen }\end{array}$ & $\begin{array}{l}\text { Phosphate } \\
(6.8)\end{array}$ & -- & -- & Chemical activation & 9 \\
\hline $\begin{array}{l}\text { D. desulfuricans } \\
\text { Ni-rubredoxin }\end{array}$ & {$\left[\mathrm{Ru}(\mathrm{bpy})_{3}\right]^{2+}$} & diffusion & ascorbate & $\begin{array}{l}\text { Phosphate } \\
(6.5)\end{array}$ & 32 & 1 & & 10 \\
\hline Co-myoglobin & {$\left[\mathrm{Ru}(\mathrm{bpy})_{3}\right]^{2+}$} & diffusion & ascorbate & $\begin{array}{l}\text { Phosphate } \\
\text { (7) }\end{array}$ & 520 & 8 & $\begin{array}{l}\text { Co-protoporphyrin IX } \\
\text { incorporated within } \\
\text { the myoglobin } \\
\text { scaffold. }\end{array}$ & 11 \\
\hline
\end{tabular}




\begin{tabular}{|c|c|c|c|c|c|c|c|c|}
\hline Catalyst & Photosensitizer & $\begin{array}{l}\text { Catalyst- } \\
\text { photosensitizer } \\
\text { interaction }\end{array}$ & $\begin{array}{l}\text { Electron } \\
\text { donor }\end{array}$ & $\begin{array}{l}\text { Solution } \\
\text { conditions } \\
(\mathrm{pH})^{a}\end{array}$ & $\begin{array}{l}\text { TON (mol } \\
\mathrm{H}_{2} / \mathrm{mol} \\
\text { catalyst) }^{b}\end{array}$ & $\begin{array}{l}\text { Longevity } \\
\text { (hours) }^{c}\end{array}$ & Comments & Ref. \\
\hline Co-cyt $b_{562}(\mathrm{M} 7 \mathrm{~A})$ & {$\left[\mathrm{Ru}(\mathrm{bpy})_{3}\right]^{2+}$} & diffusion & ascorbate & $\begin{array}{l}\text { Phosphate } \\
\text { (7) }\end{array}$ & 310 & 8 & & 12 \\
\hline
\end{tabular}

all are in water; buffers and $\mathrm{pH}$ values indicated.

${ }^{b}$ unless noted otherwise under Comments

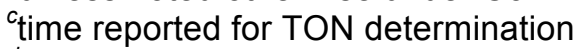

$d$ "[FeFe]" in this table indicates a diiron mimic of hydrogenase active site

${ }^{e} \mathrm{~N}, \mathrm{~N}, \mathrm{~N}$ ',N'-tetramethyl-p-phenylenediamine \& dithiothreitol

\section{Table S1 References:}

(1) Utschig, L. M.; Silver, S. C.; Mulfort, K. L.; Tiede, D. M. J. Am. Chem. Soc. 2011, 133, $16334-16337$. 13249.

(2) Silver, S. C.; Niklas, J.; Du, P.; Poluektov, O. G.; Tiede, D. M.; Utschig, L. M. J. Am. Chem. Soc. 2013, 135, 13246-

(3) Soltau, S. R.; Niklas, J.; Dahlberg, P. D.; Poluektov, O. G.; Tiede, D. M.; Mulfort, K. L.; Utschig, L. M. Chem. Commun. 2015, 51, 10628-10631.

(4) Bacchi, M.; Berggren, G.; Niklas, J.; Veinberg, E.; Mara, M. W.; Shelby, M. L.; Poluektov, O. G.; Chen, L. X.; Tiede, D.

M.; Cavazza, C.; Field, M. J.; Fontecave, M.; Artero, V. Inorg. Chem. 2014, 53, 8071-8082.

(5) Roy, A.; Madden, C.; Ghirlanda, G. Chem. Commun. 2012, 48, 9816-9818.

(6) Sano, Y.; Onoda, A.; Hayashi, T. J. Inorg. Biochem. 2012, 108, 159-162.

(7) Sano, Y.; Onoda, A.; Hayashi, T. Chem. Commun. 2011, 47, 8229-8231.

(8) Onoda, A.; Kihara, Y.; Fukumoto, K.; Sano, Y.; Hayashi, T. ACS Catal. 2014, 4, $2645-2648$.

(9) Saint-Martin, P.; Lespinat, P. A.; Fauque, G.; Berlier, Y.; Legall, J.; Moura, I.; Teixeira, M.; Xavier, A. V.; Moura, J. J. G.

Proc. Natl. Acad. Sci. U.S.A. 1988, 85, 9378-9380.

(10) Slater, J. W.; Shafaat, H. S. J. Phys. Chem. Lett. 2015, 6, 3731-3736.

(11) Sommer, D. J.; Vaughn, M. D.; Ghirlanda, G. Chem. Commun. 2014, 50, 15852-15855.

(12) Sommer, D. J.; Vaughn, M. D.; Clark, B. C.; Tomlin, J.; Roy, A.; Ghirlanda, G. Biochim. Biophys. Acta 2015, in press. DOI: 10.1016/j.bbabio.2015.09.001 